\title{
Enhancement of COVID-19 detection time by means of electrothermal force
}

\author{
Sameh Kaziz ${ }^{1,2}\left(\right.$ Yosra Saad $^{1} \cdot$ Mohamed Bouzid $^{1} \cdot$ Marwa Selmi $^{3,4} \cdot$ Hafedh Belmabrouk $^{4,5}$
}

Received: 27 March 2021 / Accepted: 5 September 2021 / Published online: 17 September 2021

(c) The Author(s), under exclusive licence to Springer-Verlag GmbH Germany, part of Springer Nature 2021

\begin{abstract}
The rapid spread and quick transmission of the new ongoing pandemic coronavirus disease 2019 (COVID-19) has urged the scientific community to looking for strong technology to understand its pathogenicity, transmission, and infectivity, which helps in the development of effective vaccines and therapies. Furthermore, there was a great effort to improve the performance of biosensors so that they can detect the pathogenic virus quickly, in reliable and precise way. In this context, we propose a numerical simulation to highlight the important role of the design parameters that can significantly improve the performance of the biosensor, in particular the sensitivity as well as the detection limit. Applied alternating current electrothermal (ACET) force can generate swirling patterns in the fluid within the microfluidic channel, which improve the transport of target molecule toward the reaction surface and, thus, enhance the response time of the biosensor. In this work, the ACET effect on the SARS-CoV-2 S protein binding reaction kinetics and on the detection time of the biosensor was analyzed. Appropriate choice of electrodes location on the walls of the microchannel and suitable values of the dissociation and association rates of the binding reaction, while maintaining the same affinity, with and without ACET effect, are also, discussed to enhance the total performance of the biosensor and reduce its response time. The two-dimensional equations system is solved by the finite element approach. The best performance of the biosensor is obtained in the case where the response time decreased by $61 \%$ with AC applying voltage.
\end{abstract}

Keywords Biosensors · COVID-19 · Rapid detection · Alternating current electrothermal (ACET) · Performance biosensor

Sameh Kaziz

kaziz_sameh@yahoo.fr

1 Quantum and Statistical Physics Laboratory, Faculty of Sciences of Monastir, University of Monastir, Environment Boulevard, 5019 Monastir, Tunisia

2 Higher National Engineering School of Tunis, Taha Hussein Montfleury Boulevard, University of Tunis, 1008 Tunis, Tunisia

3 Department of Radiological Sciences and Medical Imaging, College of Applied Medical Sciences, Majmaah University, AlMajmaah 11952, Saudi Arabia

4 Laboratory of Electronics and Microelectronics, Faculty of Science of Monastir, University of Monastir, Environment Boulevard, 5019 Monastir, Tunisia

5 Department of Physics, College of Science at Al Zulfi, Majmaah University, AlMajmaah, Saudi Arabia

\section{Introduction}

The new coronavirus, SARS-CoV-2, as named by the CSG (Gorbalenya et al. 2020), which emerged since December 2019 in Wuhan, China, has already resulted in many millions of deaths. It is so far responsible for a major pandemic with significant mortality and complete paralysis of the economies of many countries (Santiago 2020). The applications of new detection technologies are becoming increasingly important. The high affinity, specificity and sensitivity can determine the success or failure of all these detection technologies, which is fundamental to the design and operation of biosensors (Dai et al. 2011).

To find a quick solution to confront the SARS-CoV-2, scientists are using the studies on severe acute respiratory syndrome coronavirus (SARS-CoV-1) and Middle East respiratory syndrome coronavirus (MERS-CoV) which are phylogenetically close to SARS-COV-2, replicate in the pulmonary parenchyma and, like it, are responsible for a disease with potentially lateral pulmonary involvement 
(Fani et al. 2020). The coronavirus enters nasal cells with the help of its spikes protein which allows the virus to attach and then enter to human cells. After a relatively short time, 6 or $8 \mathrm{~h}$ later, the virus leaves the infected cell, usually by destroying it, and attacks its neighbors. For elderly people or those who are immunocompromised due to illness or medical treatment, the virus replicates better in such a way that the infection becomes sufficiently advanced that it is difficult to control it (Guevara-Carrion et al. 2011).

Currently, diagnostic testing for COVID-19 is based on a technique named quantitative real-time polymerase chain reaction (qRT- PCR) (Corman et al. 2020), that amplifies the RNA of SARS-CoV-2 found in infected tissue. These tests can identify tiny amounts of virus, but the method takes at least $3 \mathrm{~h}$, including the step of amplifying the viral RNA for analysis. Although real-time quantitative polymerase chain reactions are expensive, time consuming, and labor intensive (Vemula et al. 2016; Pachucki et al. 2004), due to their high sensitivity and specificity, they are considered to be the ideal standard for the design of biosensors (Choi 2020).

When false-positive or false-negative PCR results are suspected, major serological tests, including enzyme-linked immunosorbent assays (ELISA), chemiluminescent immunoassays (CLIA), and lateral flow immunoassays (LFIA), can be used to detect SARS-CoV-2 infection (Chew et al. 1256). These serological tests are useful in determining the antibody response caused by infection with SARS-CoV-2. Therefore, it is more effective in later stages (two weeks after symptoms appear) (Ghaffari et al. 2020) and cannot be used for early diagnosis. Moreover, most of these tests required trained technicians, relatively complex laboratory procedures and labeling of the sample, which might affect the activity of the antibodies.

In addition, the emerging point-of-care (POC) diagnostic technologies, which are complementary to conventional diagnostic assays, hold great promise for immunoassays in wide range of biomedical applications such as paper and textile-based biosensors. These different technologies which are used, or which are under development are based on optical, electrochemical, magnetic, and colorimetric modalities. Despite they have some disadvantages, they remain typically rapid, portable, cost effective, and especially applicable in resource-limited and developing world settings (Pashchenko et al. 2018). In this context, Kou et al. (Kou et al. 2020) have developed a smartphone-assisted metal-organic framework (MOF) biomimetic nanoreactor calorimetric paper assay allowing rapid and sensitive detection of different diseaserelated biomolecules in an easy manner. Also, choi et al. (Choi et al. 2018) proposed a new polysiloxane-modified thread-based immunoassay that provides optimal interaction between target antigen and dAb-AuNP, thereby improving device sensitivity.
The essential role of biosensors in fighting the pandemic is confirmed in last investigations (Lee et al. 2020; MoralesNarvaez and Dincer 2020; Zhu et al. 2020). However, the dependence of their performance on the surface binding reaction, which is generally limited by a slow diffusion rate and long detection time, limits their application in various situations (Nygren et al. 1987; Yang et al. 2008).

To enhance the biosensor binding reaction rate, many physical mechanisms have been applied to obtain agitation flow models in microfluidic channels such as hydrodynamic pressure (Chiu et al. 2013), AC electrokinetics (ACEK) (Yang et al. 2017; Selmi and Belmabrouk 2020), magnetic effect (Saad et al. 2021) and optical forces (Lee et al. 2000). The ACEK phenomenon mainly includes the dielectrophoresis (DEP), AC electroosmosis (ACEO), and AC electrothermal effects (ACET).

The AC electrothermal effect (ACET) was implicated in different biological technologies (Salari and Thompson 2018; Gao and Li 2019; Selmi et al. 2015, 2016a, b, 2017; Sin et al. 2010; Huang and Chang 2013). It is well known that the ACET effect is more efficient at high frequencies (above $100 \mathrm{kHz}$ ), and when having a solution with an electrical conductivity $\sigma$ that exceeds the value of $0.002 \mathrm{~S} / \mathrm{m}$ (Huang et al. 2014). Under these conditions, both experimental and numerical studies on the heterogeneous binding assays biotin/streptavidin have been achieved by Feldman et al. (2007). They have shown that electrothermal microstirring may significantly decrease nine times the reaction rate with 10 Vrms applied potential. Sigurdson et al. (2005) have also analyzed the flow of electrothermal fluid. They showed that the rate of binding reaction in heterogeneous immunoassays was enhanced seven times with an applying voltage of 6 Vrms. Huang and Chang (2013), (Huang et al. 2008) studied the kinetics of the binding reaction between the $\mathrm{C}$-reactive protein and the immunoglobulin $\mathrm{G}$. They used the two-dimensional and three-dimensional finite element method. They obtained twofold improvement in the binding reaction rate when the excitation was 15 Vrms. Selmi et al. $(2015,2016 a, b, 2017)$ have also simulated the ACET effect on the C-reactive protein binding reaction in a microfluidic biosensor. They have shown that the best position of electrodes, to get the best improvement in association and dissociation rates, is on the same wall of the microfluidic channel as the reaction surface.

Although utilizing the ACET effect for various biomedical applications has been investigated over the past two decades, more investigative work is yet to be carried out to deepen our knowledge of the phenomenon.

Recently, the numerical framework of Shahbazi et al. (2021) provided a study of the effect of three design parameters on the saturation time of biosensors that are used for the detection of coronavirus, SARS-CoV-2. The study showed that displacement of the biosensor installation position by 
only $500 \mu \mathrm{m}$ reduced detection time by more than $50 \%$. Furthermore, the increase of the input concentration has also shown a positive effect on the biosensor binding reaction. They showed that the variation of the values of the association and dissociation constants, which can be obtained by changing the ligands of the functionalized surface while maintaining the same affinity, also improved the response time of the biosensor.

Each SARS-CoV-2 virus has several structural proteins which are the surface spike (S) glycoprotein, the envelope (E) protein, the matrix $(\mathrm{M})$ protein, the nucleocapsid $(\mathrm{N})$ protein and other accessory proteins. The $\mathrm{S}$ protein maybe the most valuable biomarker for diagnosis of COVID-19 disease, it can bind to the cellular receptor, angiotensin converting enzyme 2 (ACE2) on the surface of human cells (Huang et al. 2020). The high affinity between the $S$ protein and ACE2 increases the infectivity of SARS-CoV-2.

This present study was devoted to show the influence of the ACET mechanism on the SARS-CoV-2 S protein binding reaction kinetics. The increase of the analyte mass transport, by diffusion and by convection due to the generated ACET force, increases the binding reaction rate and thus decreases the response time of the device. The peculiarity of this study, compared to that of Shahbazi et al. (2021), is that we added the effect of the ACET force on the transport mechanism and the binding reaction of SARS-CoV-2 S protein. Other authors have also introduced the ACET effect in their work, such as (Selmi et al. 2016a, b), but they have worked on other target analytes.

We have developed a two-dimensional simulation, describing the ACET effect, for a microfluidic biosensor using the finite element method. The surface complex analyte-ligand formed versus time have been also calculated.

In the first part of this work, we studied the impact of electrodes location, on the microchannel walls, on the detection time of the biosensor. Four types of geometric configurations have been investigated. In the second part, we have examined the effects of varying the association and dissociation rates, while maintaining the same biosensor affinity, with and without ACET effect, on the saturation time of the biosensor.

\section{Theoretical formulation}

The basic microfluidic biosensor has been designed as illustrated in Fig. 1 (type 1). The width, H, and the length, L, of the microchannel are $250 \mu \mathrm{m}$ and $40 \mu \mathrm{m}$, respectively.

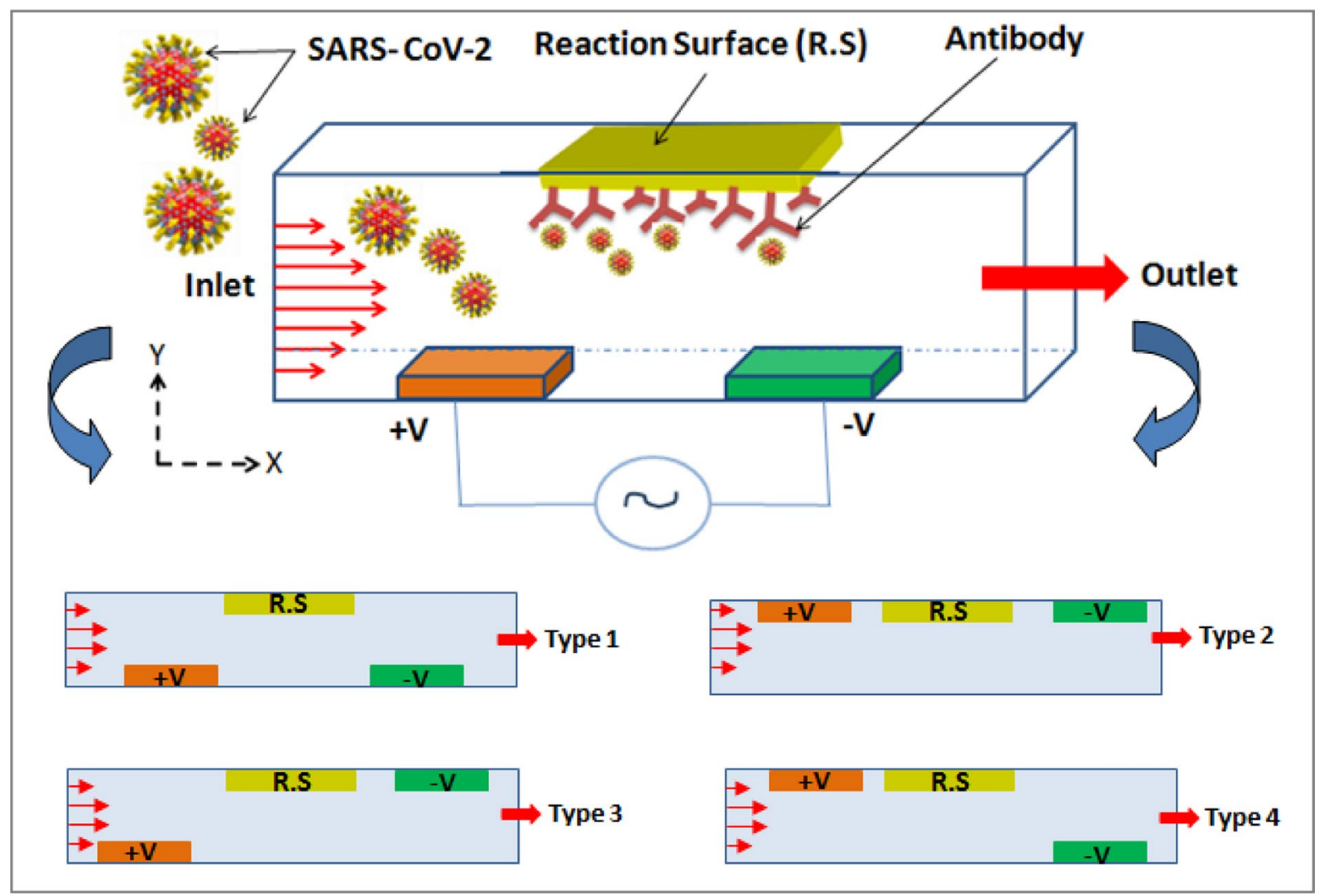

Fig. 1 Schematic model of four geometric configurations of the microfluidic biosensor with an applied external electric field 
The reaction surface, of dimensions of $20 \mu \mathrm{m}\left(\mathrm{l}_{\mathrm{S}}\right) \times 3 \mu \mathrm{m}$, is located on the top wall of the microchannel and the two electrodes, with $60 \mu \mathrm{m}$ length each $\left(\mathrm{L}_{\mathrm{E}}\right)$, are located at the bottom, just opposite to the binding surface. To look for an appropriate choice of the geometric location of the electrodes on the microfluidic channel walls, we consider three other different arrangements as presented in Fig. 1 (types 2-4).

Binding reaction is a chemical reaction between targeted analyte (A) and immobilized ligand (B) on the biosensor surface. Kinetics of this reaction is always affected by the analyte mass transport phenomenon, which is limited due to the small dimensions of the used microfluidics (order of micrometers). This, consequently, leads to a long response time of biosensors and thus limits their performances. To improve biosensors response time, fluid flow in the microfluidic channel can be accelerated to decrease the thickness of the diffusion boundary layer and improve the binding reaction of the analyte-ligand complex (AB) (Huang et al. 2008). The non-uniform electric field, applied to the fluid, generates temperature gradients due to the non-uniform joule heating. Consequently, temperature gradients induce local variations in electrical conductivity and permittivity of the fluid, responsible for begetting AC electrokinetic forces which lead to the improvement of the biosensor binding reaction rate. For this paper, we have investigated the impact of the ACET effect on the kinetics of SARS-CoV-2 S protein/antibody binding reaction. The simulation was carried out using electrostatics equation, thermal energy equation, Navier-Stokes equations, and a coupling between the second equation of Fick's law, and the first-order Langmuir adsorption model (Selmi et al. 2016a).

\subsection{Electric modeling}

The electric field $\vec{E}$ depends on the applied potential difference $\mathrm{V}$ across the electrodes and was calculated using the Poisson equation (Eq. (1)):

$\Delta V=0$ and $\vec{E}=-\nabla V$.

\subsection{Heat transfer modeling}

The fluid temperature $T$ was calculated using the thermal energy equation (Eq. (2)), where the Joule heating term, $\sigma|\vec{E}|^{2}$, is added as a source term (Soni et al. 2008). Although heating of the fluid is crucial for the ACT effect, the conduction of ambient heat helps dissipate energy so that the temperature rise in the fluid is generally low (Green et al. 2001; Ramos et al. 1998), then we can assume that the dependence of the specific heat at constant pressure $c_{p}$ and the fluid's thermal conductivity $\lambda$ versus temperature $T$ can be neglected.

$\rho C_{p} \vec{u} \cdot \nabla T=\lambda \Delta T+\sigma|\vec{E}|^{2}$.

Here, $\rho$ is the mass density, $\vec{u}$ is the velocity field, such as $(u, v)$ are its Cartesian components in two dimensions, and $\sigma$ is the fluid electrical conductivity. The viscous dissipation term has been neglected, since it is on the order of $10^{-8}$ times smaller than the Joule effect (Huang and Chang 2013).

\subsection{Laminar flow modeling}

The flow velocity field within the microfluidic channel can be solved by the two-dimensional Navier-Stokes equations, (Eqs. (3)-(4)), where $F_{e}$ represents the electrothermal force. We assumed an incompressible fluid in laminar and steadystate flow.

$\nabla \cdot \vec{u}=0$,

$\rho(\vec{u} \cdot \nabla) \vec{u}=-\nabla p+\mu \nabla^{2} \vec{u}+\overrightarrow{F_{e}}$,

where $p$ is the pressure, $\mu=10^{-3} \mathrm{~m}^{2} / \mathrm{s}$ is the kinematic viscosity of the fluid and $\rho=1000 \mathrm{Kg} / \mathrm{m}^{3}$. The fluid is transported from the channel inlet with a parabolic velocity profile (Eq. (5)):

$u(0, y)=4 u_{\max } \frac{y}{H}\left(1-\frac{y}{H}\right)$.

The velocity is zero at the wall of the channel, and gradually increases to reach the maximum value, $u_{\max }$, at the center of the channel. $H$ is the height of the microfluidic channel. Initially the fluid is assumed to be at rest.

When an external electrical field is applied to a microfluidic device, a gradient temperature is generated in the fluid due to the non-uniform Joule heating. Thus, gradients of the electrical conductivity and of the permittivity will also be generated, giving rise to the electrothermal force causing swirling velocities. The electrothermal force expression is given by (Eq. (6)):

$\overrightarrow{F_{e}}=-\frac{1}{2}\left(\frac{\nabla \sigma}{\sigma}-\frac{\nabla \varepsilon}{\varepsilon}\right) \cdot \vec{E} \frac{\varepsilon \vec{E}}{1+(\omega \tau)^{2}}-\frac{1}{4} \nabla \varepsilon|\vec{E}|^{2}$.

Where $\varepsilon$ is the relative permittivity, $\tau=\mathcal{E} / \sigma$ is the charge relaxation time of the fluid and $\omega=2 \pi v$ is the angular frequency of the electric field $\vec{E}$.

Conforming to Green et al. (2001), for water at temperature around $293 \mathrm{~K}$, we get (Eqs. (7)-(8)):

$\frac{1}{\varepsilon} \frac{\partial \varepsilon}{\partial T}=-0.004 \Rightarrow \frac{\nabla \varepsilon}{\varepsilon}=\frac{1}{\varepsilon} \frac{\partial \varepsilon}{\partial T} \nabla T=-0.004 \nabla T$, 
$\frac{1}{\sigma} \frac{\partial \sigma}{\partial T}=0.02 \Rightarrow \frac{\nabla \sigma}{\sigma}=\frac{1}{\sigma} \frac{\partial \sigma}{\partial T} \nabla T=0.02 \nabla T$.

Therefore, the electrothermal force is given by (Eq. (9)):

$$
\overrightarrow{F_{e}}=-0.012(\nabla T \cdot \vec{E}) \frac{\varepsilon \vec{E}}{1+(\omega \tau)^{2}}+0.001\left(\varepsilon|\vec{E}|^{2}\right) \nabla T .
$$

\subsection{Analyte transport and binding reaction modeling.}

The concentration of target molecules is too low and, therefore, has no effect on the viscosity of the transporter fluid (Berthier and Silberzan 2001). Using the Fick second law (Eq. (10)) and the effect of the velocity field, a complete equation to model target molecules transport can be generated (Eq. (11)):

$F=-D \nabla[A]$

$\frac{\partial[A]}{\partial t}+\vec{u} \cdot \nabla[A]=D \Delta[A]+S$,

where $F$ denotes the diffusion flux, $[A]$ is the bulk concentration of the analyte and $D$ is the diffusion constant. $S$ denotes the reaction rate. Here, $S$ is zero since there is no reaction in fluid mass.

Chemical kinetics equation of heterogeneous biosensors is modeled using the first-order Langmuir-Hinshelwood adsorption model (Berthier and Silberzan 2001; Zimmermann et al. 2005). Antibody ligands constantly trap analyte molecules to form analyte-ligand complexes and then they dissociate at a lower rate (Eq. (12)):

$\frac{\partial[A B]}{\partial t}=K_{\text {on }}\left[A_{\text {surf }}\right]\left\{\left[B_{\text {max }}\right]-[A B]\right\}-K_{\text {off }}[A B]$,

where $K_{\text {on }}$ is the association rate constant and $K_{\text {off }}$ is the dissociation rate constant, $\left[A_{\text {surf }}\right]$ is the concentration of analyte at the reaction surface, $[A B]$ is the concentration of the analyte-ligand complex at the reaction surface and $\left[B_{\max }\right]$ is the immobilized ligand concentration on the reaction surface (Sevenler et al. 2019).

Initially, the bulk analyte concentration, $[A]_{(t=0)}$, and the analyte-ligand complex, $[A B]_{(t=0)}$, were set zero.

\section{Boundary conditions}

All boundary conditions used in this model are recapped in Table 1. For the electrostatic mode, the electric potential at the electrodes was $\pm V$ and the electric insulation was applied at all other boundaries. For the heat transfer mode, the ambient temperature $T_{0}$ is applied for both electrodes, this signifies that they are isotherm with the ambient. The heat flux condition is used for the inlet and the outlet of the channel, and the other parts of the walls are allegedly thermally insulated. For laminar flow mode, the inlet fluid flows in the longitudinal direction $\mathrm{x}$ with a parabolic profile velocity, $u(0, y)$, the outlet is open to the atmosphere and all other surfaces of the microchannel are assumed to be no-slip boundaries. For the analyte transport and binding reaction mode, a constant analyte concentration $[A]_{0}$ is imported at the inlet, at the outlet the condition $\vec{n} \cdot(D \nabla[A])$ is applied. For walls and electrodes, because there is no interaction with the analyte, the homogenous Neumann condition is used (Shahbazi et al. 2021). On the sensor, there is a loss of analyte concentration due to the binding reaction with the ligand and the diffusive flux condition is imposed for this coupling between the sensor and the binding reaction.

\section{Numerical method}

Galerkin's finite element approach (Chen 2005) was applied to numerically resolve all the governing equations of the proposed model. The 2D domain has been divided into triangular geometric elements, refined near the sensitive surface and the electrodes. The convergence of our numerical calculations was confirmed after having tested different sizes of mesh grids and no serious difference appeared in the results (Aoun et al. 2016), so we used a
Table 1 Boundary conditionselectric potential, temperature, velocity and concentration for sensor, walls, electrodes, inlet, and outlet of the microfluidic channel

\begin{tabular}{|c|c|c|c|c|}
\hline Type & $\begin{array}{l}\text { Electric potential } \\
(V)\end{array}$ & $\begin{array}{l}\text { Temperature } \\
(T)\end{array}$ & $\begin{array}{l}\text { Velocity } \\
(u)\end{array}$ & $\begin{array}{l}\text { Concentration } \\
\text { (A) }\end{array}$ \\
\hline Interior & Electrostatics equation & Convection-conduction & $\begin{array}{c}\text { Navier-Stokes } \\
\text { equations }\end{array}$ & $\begin{array}{l}\text { Convection-dif- } \\
\text { fusion reaction }\end{array}$ \\
\hline Walls & Electric insulation & Thermal insulation & No slip & $\frac{\partial[A]}{\partial \eta}=0$ \\
\hline Sensor & Electric insulation & Thermal insulation & No slip & $\frac{\partial[A]}{\partial n}=-\frac{1}{D} \frac{\partial[A B]}{\partial t}$ \\
\hline Electrodes & $\pm V$ & $T_{0}$ & No slip & $\frac{\partial[A]}{\partial n}=0$ \\
\hline Inlet & Electric insulation & Heat flux & $u(0, y)$ & {$[A]_{0}$} \\
\hline Outlet & Electric insulation & Heat flux & $p=0$ & $\vec{n} \cdot(D \nabla[A])=0$ \\
\hline
\end{tabular}


number of 10,377 geometric elements. All the steps of the numerical resolution are summarized in Fig. 2. For the first step, we calculated the electric field by solving the electrostatic equation. For the second step, the Navier-Stokes equation and the thermal energy equation are solved at the same time, to obtain temperature, pressure, and velocity fields, and for the last step, the two equations of analyte transport and the binding reaction are coupled and solved together by a time dependent solver. To find out the total concentration of the formed analyte-ligand complex, we have integrated its local concentration over the entire binding surface. The dissociation curve of the binding reaction is simulated by suspending the analyte supply after the saturation time.

\section{Results and discussion}

\subsection{Validation of the numerical model}

Initially, our model was validated against literature data Berthier and Silberzan (2001). The validation is performed for a microfluidic channel with $1 \mathrm{~mm}$ in height and $10 \mathrm{~mm}$ in width. The sensor is located at the center of the bottom wall

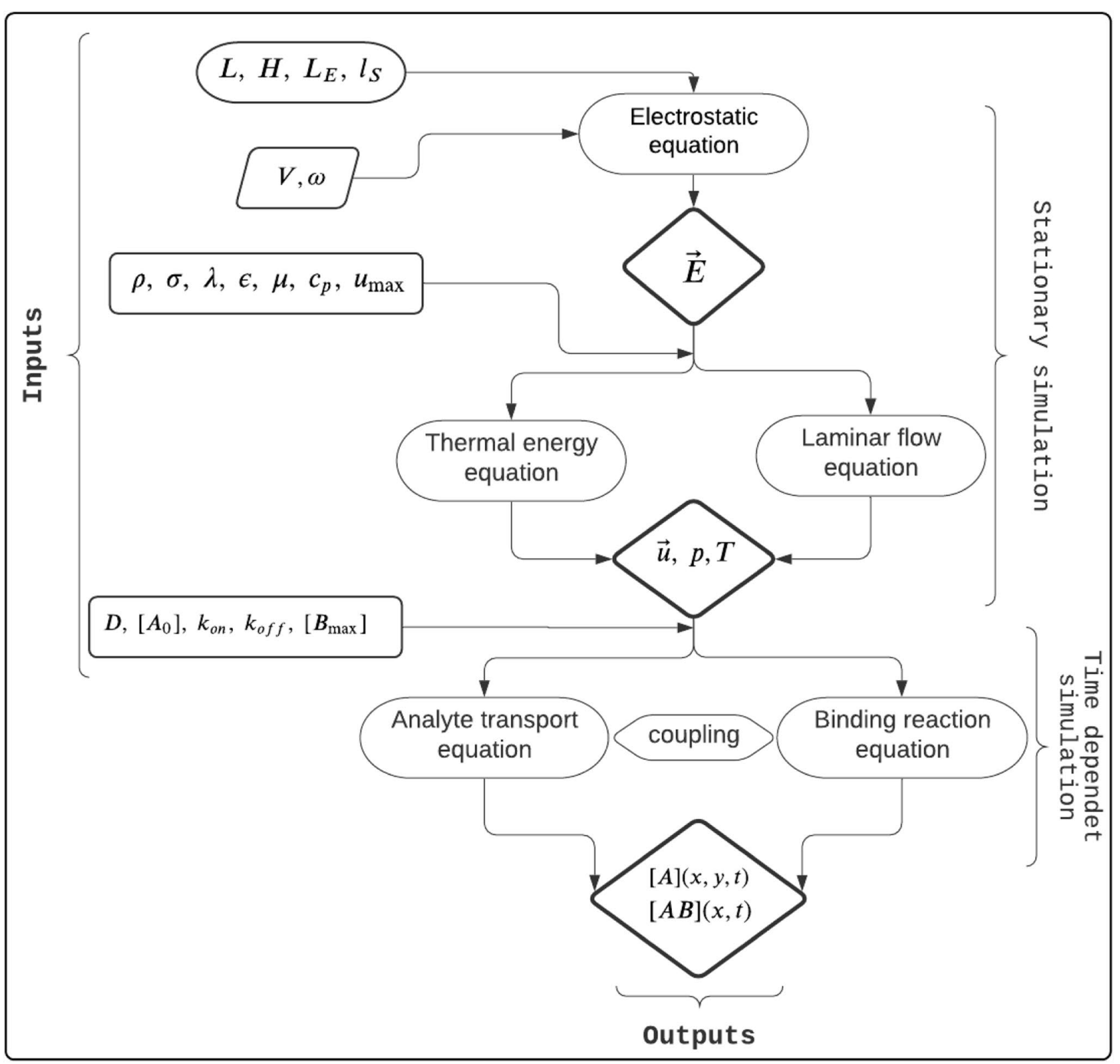

Fig. 2 Algorithm of numerical simulation-dependent steps 
of the channel. The buffer fluid flows with a flow rate equal to $10^{-6} \mathrm{~m}^{3} / \mathrm{s}$. The diffusion coefficient is $7.10^{-11} \mathrm{~m}^{2} / \mathrm{s}$ and the input concentration of analyte molecules is $2510^{-6} \mathrm{Mol} /$ $\mathrm{m}^{3}$. The density of the surface binding sites, dissociation and adsorption rates are $1668.10^{-8} \mathrm{Mol} / \mathrm{m}^{2}, 10^{-2} \mathrm{~s}^{-1}$ and $75 \mathrm{~m}^{3} /$ Mol.s respectively. Figure 3 demonstrates a well concordance between our numerical results and experimental data. The small difference between the two results is visible only in extremities.

\subsection{ACET effect on binding reaction kinetics}

After this validation step, we investigated the effect of the ACET force on the kinetics of the SARS-CoV-2 S protein/ Antibody binding reaction of the microfluidic biosensor. All parameters used for this investigation (Table 2) was taken from the previous study of Qi et al. (2006), which is a base setup designed for the detection of the coronavirus. The previous study (Qi et al. 2006) showed that, for biosensors intended for the detection of SARS-CoV (b1and h12), the antibodies affinity $\left(K_{\text {off }} / K_{\text {on }}\right)$ is in the order of $10^{-6} \mathrm{Mol} / \mathrm{m}^{3}$. Specifically, the order of magnitude of association rate is $10^{3} \mathrm{~m}^{3} / \mathrm{Mol} . \mathrm{s}$ and that for dissociation rate is $10^{-3} \mathrm{~s}^{-1}$.

In the first part of this paper, the geometry and dimensions of the used microfluidic-integrated biosensor are as illustrated in Fig. 1 (type 1). At the input, the maximum speed of the parabolic velocity, $u_{\max }$, is equal to $150 \mu \mathrm{m} / \mathrm{s}$, the applied voltage is $15 \mathrm{Vrms}$ and the frequency is $150 \mathrm{kHz}$.

The temporal evolution of the normalized surface concentration for both studies (i.e., with and without ACET effect) was plotted in Fig. 4. As illustrated, a strong improvement in

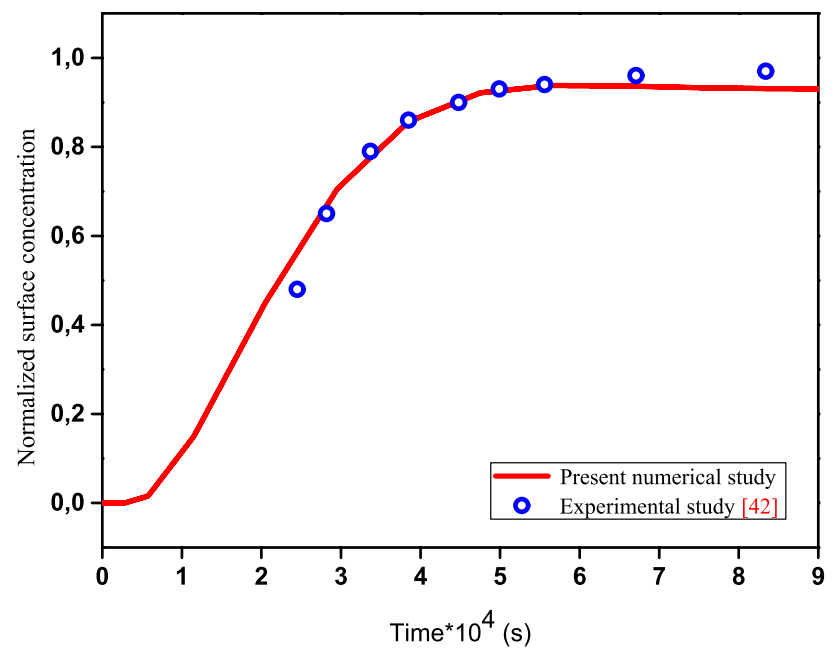

Fig. 3 Validation of the present numerical model against the experimental study of Berthier and Silberzan, (Berthier and Silberzan $2001)$, the normalized surface concentration, $[\overline{A B}]$, over time. The surface concentration is normalized to the density of binding sites on the binding surface
Table 2 SARS-CoV-2 S protein/Antibody binding parameters

\begin{tabular}{lll}
\hline Constant & Name & Value \\
\hline$K_{\text {on }}\left(\mathrm{m}^{3} / \mathrm{Mol} . \mathrm{s}\right)$ & Association rate constant & $10^{3}$ \\
$K_{\text {off }}\left(\mathrm{s}^{-1}\right)$ & Dissociation rate constant & $10^{-3}$ \\
$D\left(\mathrm{~m}^{2} / \mathrm{s}\right)$ & Diffusion constant & $10^{-11}$ \\
{$\left[B_{\max }\right]\left(\mathrm{Mol} / \mathrm{m}^{2}\right)$} & Surface ligand concentration & $3,3.10^{-8}$ \\
{$[A]_{0}\left(\mathrm{Mol} / \mathrm{m}^{3}\right)$} & Analyte input concentration & $1.10^{-11}$ \\
\hline
\end{tabular}

the binding reaction is observed in the case where the electric field is applied. This is the main reason of the ACET efficiency in boosting the immunoassay of biosensors. Indeed, the electrothermal flow engendered vortices, thereby agitating the flow, which improves the analyte mass transport toward the reaction surface and decreasing the thickness of the diffusion boundary layer.

Figure 5 shows the distribution of the flow lines for the two studies (with and without ACET effect). It can be noticed that without the excitation of the electric field, the flow structure is perfectly unidirectional with a parabolic profile, and all the current lines are parallel to the longitudinal axis of the microchannel. However, with the presence of the electric field, closed circular vortices appear, near the area between the electrodes, which are responsible for the agitation of the fluid that improves the binding reaction rate of the biosensor.

To evaluate the ACET effect on the kinetics of the binding reaction, we noted the detection time of the biosensor for both studies. Results are listed on Table 3. We can notice the decrease in the saturation time of the biosensor during the application of the electric field. Therefore, the ACET force

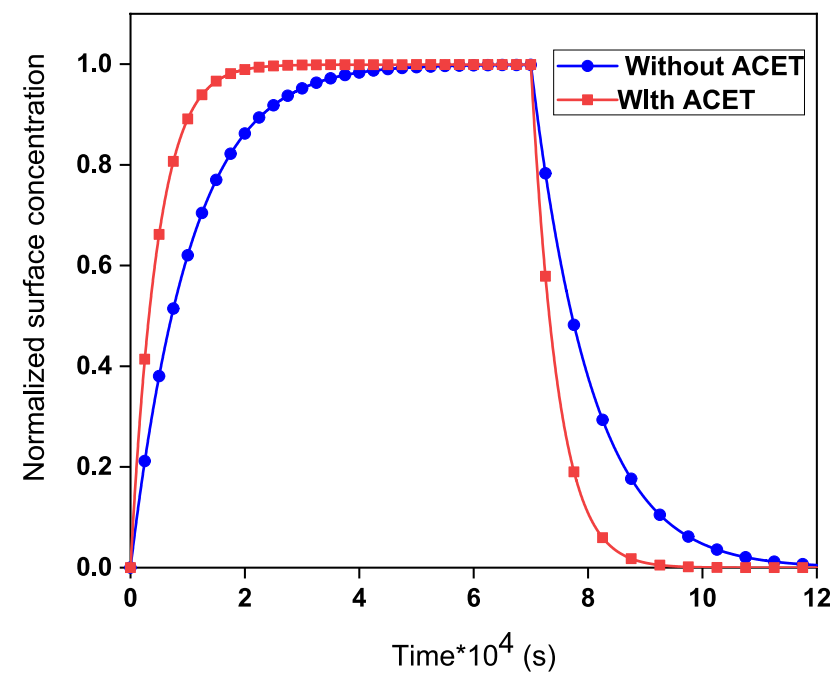

Fig. 4 Temporal evolution of the normalized surface concentration $[\overline{A B}]$ with or without applying voltage 
Fig. 5 Velocity field and flow lines (a) without ACET effect and (b) with the ACET effect

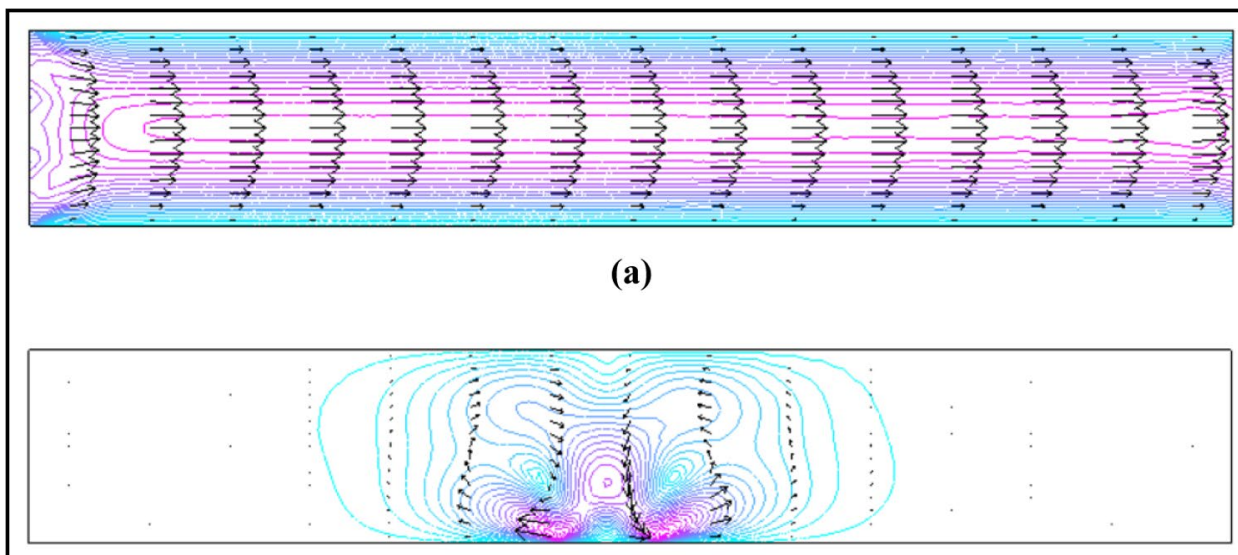

(b)
Table 3 Detection time for the binding reaction with or without ACET

\begin{tabular}{ll}
\hline Case & Detection time (s) \\
\hline Without ACET effect & 44,900 \\
With ACET effect & 20,300 \\
\hline
\end{tabular}

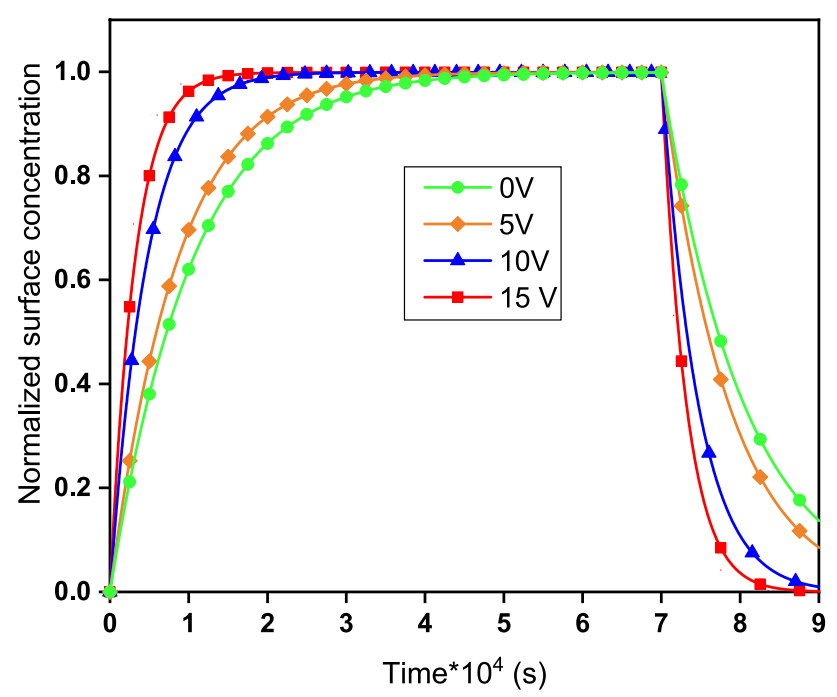

Fig. 6 Effect of various voltages on the analyte-ligand binding reaction

improves the kinetics of analyte-ligand binding reaction and, thus, the performance of the biosensor.

Then, to better understand how the response time of the biosensor varies with the excitation voltage, we successively tested voltages of 5, 10 and $15 \mathrm{~V}$ as shown in Fig. 6. It can be inferred that the enhancement of the binding reaction is relative to the voltage increase. Also, we can note that the response time of the biosensor was reduced by rising
Table 4 Biosensor response time, reduction percentage and temperature rise for different applied voltages

\begin{tabular}{llll}
\hline $\begin{array}{l}\text { Applied voltage } \\
(\mathrm{V})\end{array}$ & $\begin{array}{l}\text { Detection time } \\
(\mathrm{s})\end{array}$ & $\begin{array}{l}\text { Reduction per- } \\
\text { centage (\%) }\end{array}$ & $\begin{array}{l}\text { Tem- } \\
\text { perature } \\
\text { rise } \\
(\mathrm{K})\end{array}$ \\
\hline $5 \mathrm{~V}$ & 35,700 & 20 & 1.2 \\
$10 \mathrm{~V}$ & 20,300 & 55 & 4.8 \\
$15 \mathrm{~V}$ & 14,000 & 69 & 10.8 \\
\hline
\end{tabular}

the applied voltage (Table 4) and the reduction percentage started slowing down after a voltage of $10 \mathrm{~V}$, which can be considered as an optimum ACET voltage. The optimum voltage is the lowest possible value that still ensures functionality of biosensors because the excessive increase in the applied voltage leads to an increase in the electrothermal force, and therefore, in the fluid velocity which can harm the nature of fluids and hinder the proper functioning of detection devices.

For $10 \mathrm{~V}$ applied voltage, the temperature rise in the fluid is still low $(\Delta T<5 K)$, renders the change of fluid properties and electric field with temperature insignificant (Green et al. 2001; Ramos et al. 1998; Liu et al. 2011; Yuan et al. 2014), as supposed in such model.

\subsection{Effect of electrodes location on the binding reaction}

To look for a convenient choice of the geometric location of electrodes on the walls of the microfluidic channel, different types of arrangements are considered.

Figure 7 shows the four curves corresponding to the binding reaction for each configuration of the microfluidic biosensor shown in Fig. 1, which are excited with an optimal voltage of $10 \mathrm{Vrms}$. The temporal evolution of the 


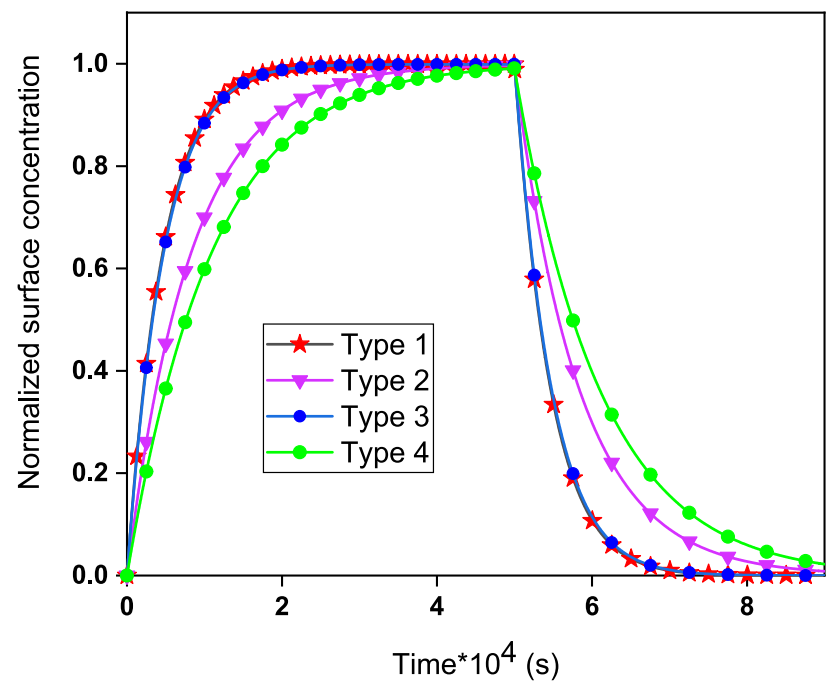

Fig. 7 Normalized surface concentration for the four biosensor arrangements excited by the optimum voltage $(10 \mathrm{~V})$ associations and dissociation phases is different for the four configuration types. The biosensor response time for type 1, and second for type 3 , is faster than for other types. This can be explained by the fact that the position of the electrodes in front of the reaction surface, can generate the most important electrothermal force, compared to the other types, causing a great agitation of the fluid which accelerates much more the mass transport of the target analyte to the biosensor reaction surface.

To justify our findings, we have plotted in Fig. 8 the velocity fields and the current lines for the four types of configurations. As illustrated, the four types show that the fluid flow contains asymmetric closed vortex patterns induced by the electrothermal effect. The position, number and size of these vortices depend on the location of the electrodes on the microchannel walls. We can note, also that in all configuration types, the greatest value of the flow velocity occurs in a small region very close to the electrodes and, as expected, the highest velocity value is found for type 1 which is around $3.7 \mathrm{~mm} / \mathrm{s}$.
Fig. 8 Velocity field and flow lines with ACET effect for the four configuration types

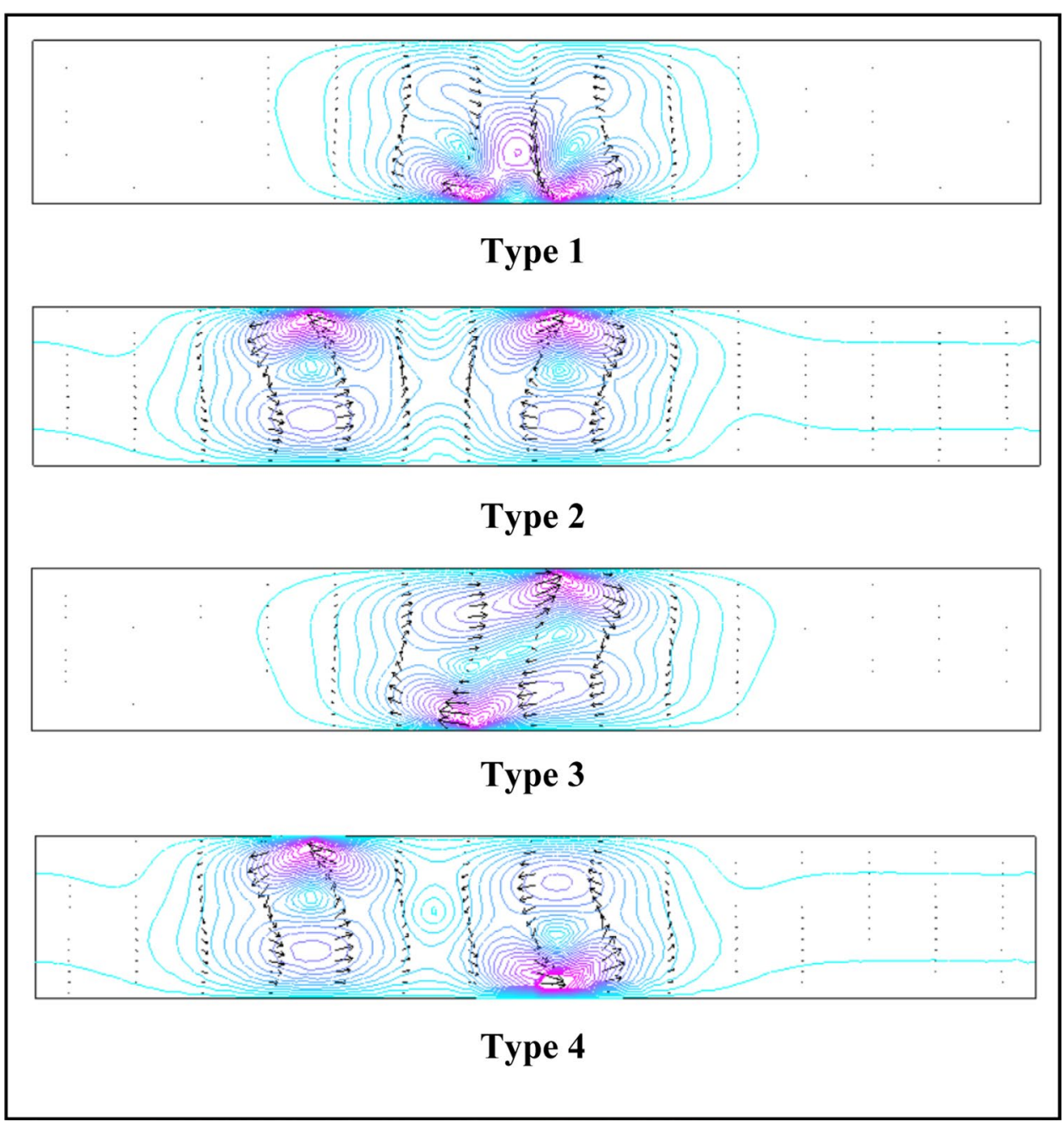


Table 5 Saturation time and reduction percentage for the four types of microfluidic biosensors under ACET effect

\begin{tabular}{lll}
\hline Configuration & Detection time (s) & $\begin{array}{l}\text { Reduction } \\
\text { percentage } \\
(\%)\end{array}$ \\
\hline Type 1 & 20,300 & 55 \\
Type 2 & 39,325 & 12 \\
Type 3 & 20,875 & 53 \\
Type 4 & 41,575 & 7 \\
\hline
\end{tabular}

Table 5 shows the detection time and the reduction percentage for each configuration type. We note, as expected, that the largest enhancement values are observed for type 1. It is also noted that the electrothermal force is more powerful and similarly improves the binding reaction for type 1 and type 3 than other types.

\subsection{Effect of the ligands on the binding reaction kinetics (with or without ACET effect)}

The kinetics of heterogeneous immunoassays depends on the values of association and dissociation rates of the analyte-ligand trapping reaction. These rates can vary by changing the type of ligands on the binding surface while maintaining the same affinity. In this part of the paper, we studied the ACET effect on the binding reaction of three sensors, having the same affinity but different amplitudes of adsorption and desorption rates. We used one pair of electrodes, located as in configuration of type 1 . Figure 9 shows the results of three cases in which the adsorption and dissociation rates have been changed, while retaining the same affinity, with and without ACET. As the association and

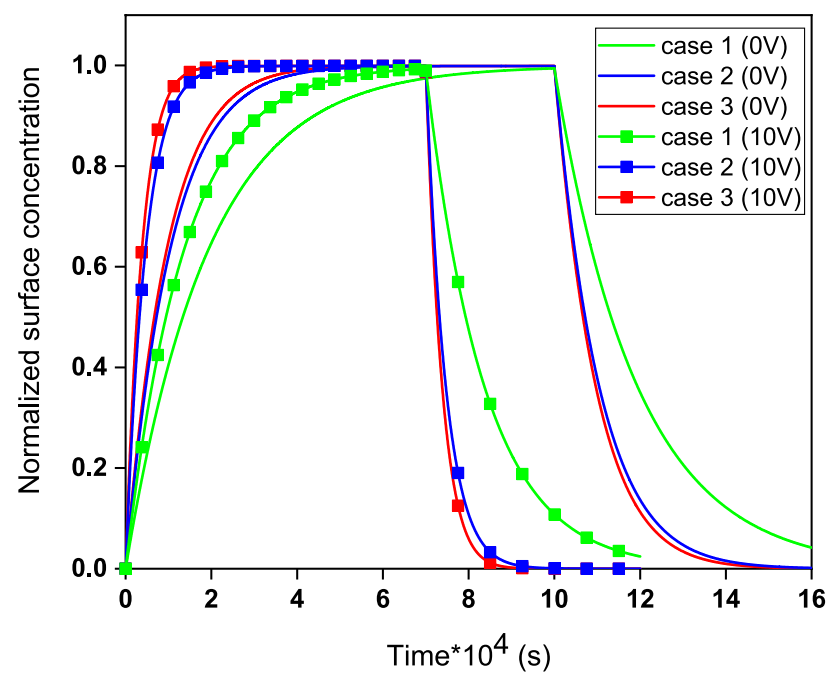

Fig. 9 ACET effect on binding reaction of three sensors with different reaction rates dissociation rates increase, the response time of the biosensor decrease. In addition, saturation time shows an important increase in the case when the association and dissociation rates respectively attain orders of $10^{2} \mathrm{~m}^{3} / \mathrm{Mol}$.s and $10^{-4} 1 / \mathrm{s}$ (case 1 in both studies).

The results obtained with the current model, without ACET effect, have the same behavior as the simulation results of Shahbazi et al. (2021). With ACET effect, the saturation time more decreases in the three cases, thus reducing remarkably the response time of the biosensor.

Also, we have calculated the reduction percentage of the response time of the biosensor for the three cases with ACET effect, compared to the same cases without ACET effect. Obtained results are shown in the Table 6. We can note the important decrease of the saturation time of the biosensor under ACET effect, especially in case 3 where the saturation time decrease by $61 \%$ with ACET effect.

\section{Conclusion}

This work has highlighted the importance of the ACET effect in improving the performance of biosensors intended for COVID-19 detection. The ACET force generated by an applied electric field was an efficient way to transport the analyte molecules toward the binding surface of the biosensor and reduce dramatically its response time. Some parameters, such as the applied voltage, the location of electrodes on the microchannel walls and the change of the binding reaction rates have been investigated. The results showed that a great enhancement of the binding reaction of a biosensor appears when a pair of electrodes was placed on the bottom wall of the microchannel, in front of the reaction surface. In this case, the reduction percentage is about 55\%, when the optimal voltage was applied. In addition, it was found that the variation of the association and dissociation rates under ACET effect may decrease the saturation time of the biosensor by $61 \%$ compared to the same case without applying voltage.

Table 6 Response time and reduction percentage of three sensors having different association and dissociation rates, with and without ACET effect

\begin{tabular}{llll}
\hline Case & $\begin{array}{l}K_{\text {on }} \\
{\left[\mathrm{m}^{3} / \mathrm{Mol} . \mathrm{s}\right],} \\
K_{\text {off }}[1 / \mathrm{s}]\end{array}$ & Detection time (s) & $\begin{array}{l}\text { Reduction } \\
\text { percentage } \\
(\%)\end{array}$ \\
\hline Case 1 $(0 \mathrm{~V})$ & $10^{2}, 10^{-4}$ & 88,475 & $/$ \\
Case 2 $(0 \mathrm{~V})$ & $10^{3}, 10^{-3}$ & 44,900 & $/$ \\
Case 3 $(0 \mathrm{~V})$ & $10^{4}, 10^{-2}$ & 40,600 & $/$ \\
Case 1 $(10 \mathrm{~V})$ & $10^{2}, 10^{-4}$ & 63,325 & 28 \\
Case 2 $(10 \mathrm{~V})$ & $10^{3}, 10^{-3}$ & 20,300 & 55 \\
Case 3 $(10 \mathrm{~V})$ & $10^{4}, 10^{-2}$ & 15,875 & 61
\end{tabular}


Current work is geared towards creating ingenious designs for future biosensors that can help reduce the risks of potential other waves or strains of SARS-CoV-2. Future work could be related to the development of a new kinetic adsorption model and even this work could be complemented by an experimental exploration. We can also, combine effects of DEP and ACET techniques to better improve diffusive transport and the mixing performance.

Author contributions SK: Conceptualization, methodology, data curation. YS: Ressources, validation. MB: Writing-original draft preparation. MS: Project administration, visualization. HB: investigation, supervision.

\section{Declarations}

Conflict of interest The authors declare that they have no known competing financial interests or personal relationships that could have appeared to influence the work reported in this paper.

\section{References}

Aoun N, Echouchene F, Diallo AK, Launay J, Belmabrouk H (2016) Finite-element simulations of the pH-ElecFET microsensors. IEEE Sensors J 16(17):6519-6526

Berthier J, Silberzan P (2001) Microfluidics for biotechnology. ARTECH HOUSE, Boston, London

Chen Z (2005) Finite element methods and their applications (scientific computation). Springer-Verlag, Berlin, Germany

Chew KL, Tan SS, Saw S, Pajarillaga A, Zaine S, Khoo C, Wang W, Tambyah P, Jureen R, Sethi SK (2020) Clinical evaluation of serological IgG antibody response on the Abbott Architect for established SARS-CoV-2 infection. Clin Microbiol Infect 26(9):1256. E9-1256.E11

Chiu YJ, Cho SH, Mei Z, Lien V, Wu TF, Lo YH (2013) Universally applicable three-dimensional hydrodynamic microfluidic flow focusing. Lab Chip 13:1803

Choi JR (2020) Development of point-of-care biosensors for COVID19. Front Chem 8:517

Choi JR, Nilghaz A, Chen L, Chou KC, Lu X (2018) Modification of thread-based microfluidic device with polysiloxanes for the development of a sensitive and selective immunoassay. Sens Actuators, B Chem 260:1043-1051

Corman VM, Landt O, Kaiser M (2020) Detection of 2019 novel coronavirus (2019-nCoV) by real-time RT-PCR. Euro Surveill 25(3):2000045

Dai TL, Nguyen BH, Van Hieu N, Tran HV, Le Nguyen H, Nguyen PX (2011) Electrochemical detection of short HIV sequences on chitosan/Fe3O4 nanoparticle based screen printed electrodes. Mater Sci Eng: C 31(2):477-485

Fani M, Teimoori A, Ghafari S (2020) Comparison of the COVID-2019 (SARS-CoV-2) pathogenesis with SARS-CoV and MERS-CoV infections. Future Virol 15(5):317-323

Feldman HC, Sigurdson M, Meinhart CD (2007) AC electrothermal enhancement of heterogeneous assays in microfluidics. Lab Chip $7: 1553-1559$

Gao X, Li Y (2019) Simultaneous microfluidic pumping and mixing using an array of asymmetric 3D ring electrode pairs in a cylindrical microchannel by the AC electroosmosis effect. Eur J Mech B/ fluids 75:361-371
Ghaffari A, Meurant R, Ardakani A (2020) COVID-19 Serological Tests: How Well Do They Actually Perform. Diagnostics 10(7):453

Gorbalenya A, Baker S, Baric R, de Groot R, Drosten C, Gulyaeva A, Haagmans B, Lauber C, Leontovich A, Neuman B, Penzar D, Perlman S, Poon L, Samborskiy D, Sidorov I, Sola I, Ziebuhr J (2020) The species severe acute respiratory syndrome-related coronavirus: classifying $2019-\mathrm{nCoV}$ and naming it SARSCoV-2. Nat Microbiol 5:536-544

Green NG, Ramos A, González A, Castellanos A, Morgan H (2001) Electrothermally induced fluid flow on microelectrodes. J Electrostat 53:71-87

Guevara-Carrion G, Hasse H, Vrabec J (2011) Thermodynamic properties for applications in chemical industry via classical force fields. Multiscale molecular methods in applied chemistry. Springer, Berlin, Heidelberg, pp 201-249

Huang K-R, Chang J-S (2013) Three-dimensional simulation on binding efficiency of immunoassay for a biosensor with applying electrothermal effect. Heat Mass Transf 49:1647-1658

Huang K-R, Chang JS, Chao SD, Wu KC, Yang CK, Lai CY, Chen SH (2008) Simulation on binding efficiency of immunoassay for a biosensor with applying electrothermal effect. J Appl Phys 104:064702

Huang Y-H, Chang JS, Chao SD, Wu KC, Huang L (2014) Improving the binding efficiency of quartz crystal microbalance biosensors by applying the electrothermal effect. Biomicrofluidics 8:054116

Huang Y, Yang C, Xu Xf, Xu W, Liu SW (2020) Structural and functional properties of SARS-CoV-2 spike protein: potential antivirus drug development for COVID-19. Acta Pharmacol Sin 41:1141-1149

Kou X, Tong L, Shen Y, Zhu W, Yin L, Huang S, Zhu F, Chen G, Ouyang G (2020) Smartphone-assisted robust enzymes@ MOFs-based paper biosensor for point-of-care detection. Biosens Bioelectron 156:112095

Lee GU, Metzger S, Natesan M, Yanavich C, Dufrêne YF (2000) Implementation of force differentiation in the immunoassay. Anal Biochem 287:261-271

Lee HB, Meeseepong M, Trung TQ, Kim BY, Lee NE (2020) A wearable lab-on-a-patch platform with stretchable nanostructured biosensor for non-invasive immunodetection of biomarker in sweat. Biosens Bioelectron 156:112133

Liu X, Yang K, Wadhwa A, Eda S, Li S, Wu J (2011) Development of an AC electrokinetics-based immunoassay system for onsite serodiagnosis of infectious diseases. Sens Actuator A Phys 171:406-413

Morales-Narvaez E, Dincer C (2020) The impact of biosensing in a pandemic outbreak: covid-19. Biosens Bioelectron 163:112274

Nygren HK, Werthen M, Stenberg M (1987) Kinetics of antibody binding to solid-phase-immobilised antigen: effect of diffusion rate limitation and steric interaction. J Immunol Methods 101:63-71

Pachucki CT, Khurshid MA, Nawrocki J (2004) Utility of reverse transcriptase PCR for rapid diagnosis of influenza a virus infection and detection of amantadine-resistant influenza a virus isolates. J Clin Microbiol 42:2796-2798

Pashchenko O, Shelby T, Banerjee T, Santra S (2018) A comparison of optical, electrochemical, magnetic, and colorimetric point-ofcare biosensors for infectious disease diagnosis. ACS Infect Dis 4(8):1162-1178

Qi C, Duan JZ, Wang ZH, Chen YY, Zhang PH, Zhan L, Yan XY, Cao WC, Jin G (2006) Investigation of interaction between two neutralizing monoclonal antibodies and SARS virus using biosensor based on imaging ellipsometry. Biomed Microdevices 8:247-253

Ramos A, Morgan H, Green NG, Castellanos A (1998) Ac electrokinetics: a review of forces in microelectrode structures. J Phys D Appl Phys 31:2338-2353 
Saad Y, Selmi M, Gazzah MH, Belmabrouk H (2021) The magnetic field effect on the improvement of the binding reaction of C-reactive protein at the microfluidic channel surface of an SPR biosensor. Eur Phys J plus 136:608

Salari A, Thompson M (2018) Recent advances in AC electrokinetic sample enrichment techniques for biosensor development. Sens Actuators B Chem 255:3601-3615

Santiago I (2020) Trends and innovations in biosensors for covid-19 mass testing. ChemBioChem 21:2880-2889

Selmi M, Belmabrouk H (2020) AC electroosmosis effect on microfluidic heterogeneous immunoassay efficiency. Micromachines $11: 342$

Selmi M, Echouchene F, Gazzah MH, Belmabrouk H (2015) Flow confinement enhancement of heterogeneous immunoassays in microfluidics. IEEE Sens J 15:7321-7328

Selmi M, Gazzah MH, Belmabrouk H (2016a) Numerical study of the electrothermal effect on the kinetic reaction of immunoassays for a microfluidic biosensor. Langmuir 32:13305-13312

Selmi M, Khemiri R, Echouchene F, Belmabrouk H (2016b) Electrothermal effect on the immunoassay in a microchannel of a biosensor with asymmetrical interdigitated electrodes. Appl Therm Eng 105:77-84

Selmi M, Gazzah MH, Belmabrouk H (2017) Optimization of microfluidic biosensor efficiency by means of fluid flow engineering. Sci Rep 7:1-11

Sevenler D, Trueb J, Unlü MS (2019) Beating the reaction limits of biosensor sensitivity with dynamic tracking of single binding events. Proc Natl Acad Sci USA 116:4129-4134

Shahbazi F, Jabbari M, Esfahani MN, Keshmiri A (2021) A computational simulation platform for designing real-time monitoring systems with application to COVID-19. Biosens Bioelectron $171: 112716$
Sigurdson M, Wang D, Meinhart CD (2005) Electrothermal stirring for heterogeneous immunoassays. Lab Chip 5:1366-1373

Sin ML, Gau V, Liao JC, Wong PK (2010) Electrothermal fluid manipulation of high-conductivity samples for laboratory automation applications. J Assoc Lab Autom 15:426-432

Soni G, Singurdson M, Meinhart C (2008) AC electrokinetically enhanced surface reaction. Model Libr MEMS Module COMSOL Multiphys V3 5a:220-247

Vemula SV, Zhao J, Liu J, Wang X, Biswas S, Hewlett I (2016) Current approaches for diagnosis of influenza virus infections in humans. Viruses 8:96

Yang CK, Chang JS, Chao SD, Wu KC (2008) Effects of diffusion boundary layer on reaction kinetics of immunoassay in a biosensor. J Appl Phys 103:1160-1169

Yang K, Islam N, Eda S, Wu J (2017) Optimization of an ac electrokinetics immunoassay lab-chip for biomedical diagnostics. Microfluid Nanofluid 21:35

Yuan Q, Yang K, Wu J (2014) Optimization of planar interdigitated microelectrode array for biofluid transport by AC electrothermal effect. Microfluid Nanofluid 16:167-178

Zhu H, Fohlerova Z, Pekarek J, Basova E, Neu`zil P (2020) Recent advances in lab- on-a-chip technologies for viral diagnosis. Biosens Bioelectron 153:112041

Zimmermann M, Delamarche E, Wolf M, Hunziker P (2005) Modeling and optimization of high-sensitivity, low-volume microfluidicbased surface immunoassays. Biomed Microdevices 7:99-110

Publisher's Note Springer Nature remains neutral with regard to jurisdictional claims in published maps and institutional affiliations. 